\title{
El Premio de Investigación José Félix Patiño Restrepo, promotor de la investigación en nutrición en Colombia
}

\author{
The Restrepo award: promoting nutrition \\ research in Colombia
}

Fanny Aldana-Parra, ND, Esp, cPhD. ${ }^{1}$

https://doi.org/10.35454/rncm.v1n2.049

La investigación científica surge de la necesidad del ser humano de encontrar explicación a los fenómenos observados; es una práctica inherente a la curiosidad del hombre por aprender de su entorno y mejorarlo, así como alcanzar un conocimiento seguro que permita estar preparado para actuar. Y aunque en la actualidad existe una crítica posmodernista a la ciencia con respecto a los valores sobre los cuales se desarrolla el nuevo conocimiento, lo cierto es que la investigación científica ha evolucionado desde el modelo clásico inspirado en Aristóteles ${ }^{(1,2)}$ hasta un modelo en el cual el conocimiento debe ser probado, validado, refutado, difundido y replicado. Se entiende entonces la investigación científica como el motor de desarrollo e innovación de un país, generadora de conocimiento e impulsora de la competitividad científica y económica de una región. De tal forma que, los países con mayor inversión en ciencia, investigación y desarrollo, cuentan con los coeficientes de invención más altos. Un ejemplo claro en Latinoamérica es Brasil, en donde la inversión en Investigación y Desarrollo fue de US\$23.003 millones en 2015, lo cual representa el 1,27 \% del Producto Interno Bruto (PIB) y un coeficiente de innovación de
3,59, cifra no muy lejana a la lograda por países como Corea durante este mismo año (coeficiente de innovación de 4). En Colombia, la inversión en ciencia y tecnología en el año 2006 fue US\$ de 241.62 millones, cifra que ascendió a US\$1.140,18 millones en 2014 (0,3\% del PIB), con un descenso a US $\$ 838,4$ millones en 2015 (0,271 \% del PIB), lo cual representó en este último año un coeficiente de invención de $0,67^{(3,4)}$.

Aunque $70 \%$ de la investigación en Colombia se desarrolla dentro de las universidades, la situación de la investigación en Colombia hace necesario establecer incentivos que motiven a los profesionales de la salud a iniciar o continuar sus proyectos de investigación y socializarlos dentro de la comunidad científica. Por tal motivo, la Asociación Colombiana de Nutrición Clínica, dentro de los objetivos contemplados en el Artículo 7 de sus estatutos, ha apoyado la investigación en temas relacionados con nutrición y soporte nutricional durante 29 años, mediante el Premio de Investigación José Félix Patiño Restrepo. En esta oportunidad (2018), se contó con la participación de 44 trabajos libres, trabajos de grado y protocolos de investigación, alcanzando la convocatoria más concurrida en la historia del premio. Tanto

1 Investigadora Pontificia Universidad Javeriana, Departamento de Nutrición y Bioquímica, Bogotá D.C., Colombia.

Coordinadora del Premio José Félix Patiño Restrepo, ACNC. 
investigadores reconocidos, estudiantes de pregrado que inician sus carreras, como investigadores de diferentes instituciones del país, dieron cuenta del creciente interés de la comunidad científica colombiana interesada en temas de nutrición, por aportar conocimiento en esta área. El apoyo a los investigadores y la divulgación del conocimiento adquirido en el desarrollo de protocolos de investigación en nutrición y en la práctica clínica nutricional son los pilares fundamentales de esta iniciativa. El futuro del Premio de investigación José Félix Patiño Restrepo es promisorio y el apoyo brindado por la Asociación Colombiana de Nutrición clínica ya está dando sus frutos.

\section{Referencias bibliográficas}

1. Tredennick H. Aristotle: posterior analytics. Londres: Loeb Classical Library; 1960.

2. Peirce CS. The fixation of belief. Princeton University Press; 2009.

3. Albornoz M. El estado de la ciencia: Principales Indicadores de Ciencia y Tecnología Iberoamericanos / Interamericanos 2017. Buenos Aires: Altuna Impresores; 2017.

4. Lucio J. Indicadores de ciencia y tecnología. Bogotá: Observatorio Colombiano de Ciencia y Tecnología; 2016. 